Corrigendum

\title{
Corrigendum to "Instrumentation for diagnostics and control of laser-accelerated proton (ion) beams” [Phys Med 30 (2014) 255-270]
}

\section{P.R. Bolton ${ }^{\mathrm{a}, *}$, M. Borghesi ${ }^{\mathrm{b}}$, C. Brenner ${ }^{\mathrm{c}}$, D.C. Carroll ${ }^{\mathrm{c}}$, C. De Martinis ${ }^{\mathrm{d}}$, F. Fiorini ${ }^{\mathrm{e}}$,} A. Flacco ${ }^{\text {f }}$, V. Floquet ${ }^{\text {f }}$, J. Fuchs ${ }^{\text {f }}$, P. Gallegos ${ }^{\text {g }}$, D. Giove ${ }^{\text {d }}$, J.S. Green ${ }^{\text {c }}$, S. Green ${ }^{\text {h }}$, B. Jones ${ }^{\text {i }}$, D. Kirby ${ }^{\text {h}}$, P. McKenna ${ }^{g}$, D. Neely ${ }^{\text {c}}$, F. Nuesslin ${ }^{\text {j}}$, R. Prasad ${ }^{\text {b }}$, S. Reinhardt ${ }^{\text {k}}$, M. Roth ${ }^{1}$, U. Schramm ${ }^{\mathrm{m}}$, G.G. Scott ${ }^{\mathrm{g}}$, S. Ter-Avetisyan ${ }^{\mathrm{b}}$, M. Tolley ${ }^{\mathrm{c}}$, G. Turchetti ${ }^{\text {n, J.J. Wilkens }}{ }^{\mathrm{o}}$

\footnotetext{
${ }^{a}$ Kansai Photon Science Institute, Japan Atomic Energy Agency, 8-1-7 Umemidai, Kizugawa, Kyoto 619-0215, Japan

${ }^{\mathrm{b}}$ Centre for Plasma Physics, School of Mathematics and Physics, Queen's University Belfast, BT7 1NN, United Kingdom

${ }^{c}$ Central Laser Facility, STFC Rutherford Appleton Laboratory, Oxfordshire OX11 OQX, United Kingdom

d Dipartimento di Fisica, Universita di Milano and INFN Sezione di Milano, Milano, Italy

e Oxford Institute for Radiation Oncology (CRUK and MRC). University of Oxford, Oxford OX3 7DQ. United Kingdom

${ }^{\mathrm{f}}$ Laboratoire pour l'Utilisation des Lasers Intenses, Ecole Polytechnique, 91128 Palaiseau, France

g SUPA, Department of Physics, University of Strathclyde, Glasgow G4 ONG, United Kingdom

hepartment of Medical Physics, University Hospital Birmingham, Birmingham, B15 2TH, United Kingdom

${ }^{\mathrm{i}}$ Gray Institute for Radiation Oncology and Biology, University of Oxford, OX3 7DQ Oxford, United Kingdom

j Biomedical Physics, Klinikum Rechts der Isar, Technische Universität München, D-81675 München, Germany

${ }^{\mathrm{k}}$ Fakultät für Physik, Ludwig-Maximilians Universität München, 85748 Garching, Germany

${ }^{1}$ Institut fur Kernphysik, Technische Universitat Darmstadt, 64289 Darmstadt, Germany

${ }^{\mathrm{m}}$ Institute for Radiation Physics, Helmholtz-Zentrum Dresden-Rossendorf, 01314 Dresden, Germany

nepartimento di Fisica, Universita di Bologna and INFN Sezione di Bologna, Bologna, Italy

${ }^{\circ}$ Department of Radiation Oncology, Technische Universitat Munchen, Klinikum rechts der Isar, D-81675 Munchen, Germany
}

Due to an error in the manuscript submission process Dr. Francesca Fiorini of the Oxford Institute for Radiation Oncology was not included in the list of authors of the published work entitled "Instrumentation for diagnostics and control of laser-accelerated proton (ion) beams". We deeply regret the occurrence and inconvenience of this error.

This corrigendum is our published correction to the erroneous omission in which we include Dr. F. Fiorini as a listed author of this work. The correct complete list of the authors of the paper, "Instrumentation for diagnostics and control of laser-accelerated proton (ion) beams" (Phys Med 2014; 30: 255-270) is the following:

P.R. Bolton, M. Borghesi, C. Brenner, D.C. Carroll, C. De Martinis, F. Fiorini, A. Flacco, V. Floquet, J. Fuchs, P. Gallegos, D. Giove, J. S. Green, S. Green, B. Jones, D. Kirby, P. McKenna, D. Neely, F. Nuesslin, R. Prasad, S. Reinhardt, M. Roth, U. Schramm, G.G. Scott, S. Ter-Avetisyan, M. Tolley, G. Turchetti, and J.J. Wilkens

The authors would like to apologise for any inconvenience caused.

DOI of original article: http://dx.doi.org/10.1016/j.ejmp.2013.09.002

* Corresponding author. Tel: +81 77471 3455; fax: +81 774713316

E-mail addresses: bolton.paul@jaea.go.jp, boltonpr@gmail.com (P.R. Bolton). 